CASE REPORT

\title{
Chinese Traditional Cupping Therapy to Treat Fat Liquefaction at SNM Surgery - Case Report
}

\author{
Linxi Yang ${ }^{1}$, Weilin Fang ${ }^{2}$, Ying $W u^{3}$ and Jiayi Li $^{2 *}$ \\ ${ }^{1}$ Department of Gastrointestinal Surgery, Renji Hospital, School of Medicine, Shanghai Jiaotong University, China \\ ${ }^{2}$ Department of Urology, Renji Hospital, School of Medicine, Shanghai Jiaotong University, China \\ ${ }^{3}$ Department of Ultrasound, Renji Hospital, School of Medicine, Shanghai Jiaotong University, China \\ *Corresponding author: Jiayi Li, Department of Urology, Renji Hospital, School of Medicine, Shanghai \\ Jiaotong University, China
}

\section{Introduction}

Sacral neuromodulation (SNM) is a minimally invasive technique to modulate spinal reflexes that influence the bladder, bowel, sphincter, and pelvic floor to improve and restore voiding and storage functions [1]. It includes two phases of surgery and patients with symptomatic relief during stage I are permanently implanted with a sacral pulse generator. The most common adverse events are change in stimulation, implant site pain, and infection. However, there are no reports of fat liquefaction at the SNM incision site. The infection rate for SNM has been reported to range from $3 \%$ to $10 \%$ [2]. Fat liquefaction occurs commonly as a surgical complication, especially in surgery with a large abdominal incision. The causes may be related to obesity, unnecessary and overuse of electrocautery in surgery, prolonged exposure of the incision, mechanical irritation, old age and chronic disease [3]. Once the fat liquefaction occurs, incision and drainage or re-operation may be unavoidable.

Herein, we report the case of an overactive bladder (OAB) patient who developed the complication of fat liquefaction after SNM Stage II surgery. We used traditional Chinese cupping (Figure 1), to suck the liquid from the incision site instead of any interventional procedure, hoping to avoid re-operation. This non-invasive technique shows promising initial results reducing the liquefaction area, but long-term efficacy remains to be seen.

\section{Case Presentation}

A 26-year-old Chinese female whose BMI was 36.33 had a history of 2 years of urinary frequency, urgency and urge incontinence without dysuria. She voided more than 20 times a day during waking hours and awoke 5 times a night to void. She noted enuresis but had no voiding difficult.

Her urinalysis was negative and her post-void residual was $5 \mathrm{ml}$. She was diagnosed with $O A B$ and was refractory to behavioral therapy and anti-muscarinic agents. The patient was then offered Sacral Neuromodulation. She underwent a stage I lead placement on October $28^{\text {th }}, 2017$. After reporting greater than 50\% symptom improvement she underwent the stage II operation on November $17^{\text {th }}, 2017$.

On the seventh postoperative day (after the stage II surgery), the wound tissue was liquefied and broken (Figure 2). We used a Chinese traditional cupping technique to aspirate about $6 \mathrm{ml}$ of liquid two days later. A day later, the skin appeared intact, and then we began to monitor ultrasound changes without any more aspiration. Superficial ultrasound showed no obvious liquefaction at the surgical field on the second day. A small amount of liquefaction was found at the stimulator position under superficial ultrasound six days later. The liquefaction area further enlarged ten days later. Similar images appeared on an ultrasound thirteen days later. Seventeen days later, the superficial ultrasound showed reduced liquefaction at the stimulator posi-

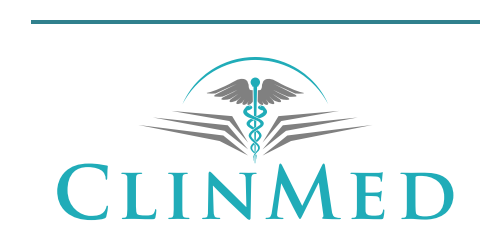

INTERNATIONAL LIBRARY
Citation: Yang L, Fang W, Wu Y, Li J (2020) Chinese Traditional Cupping Therapy to Treat Fat Liquefaction at SNM Surgery - Case Report. Int Arch Urol Complic 6:072. doi.org/10.23937/2469-5742/1510072 Accepted: May 25, 2020: Published: May 27, 2020

Copyright: (C) 2020 Yang L, et al. This is an open-access article distributed under the terms of the Creative Commons Attribution License, which permits unrestricted use, distribution, and reproduction in any medium, provided the original author and source are credited. 


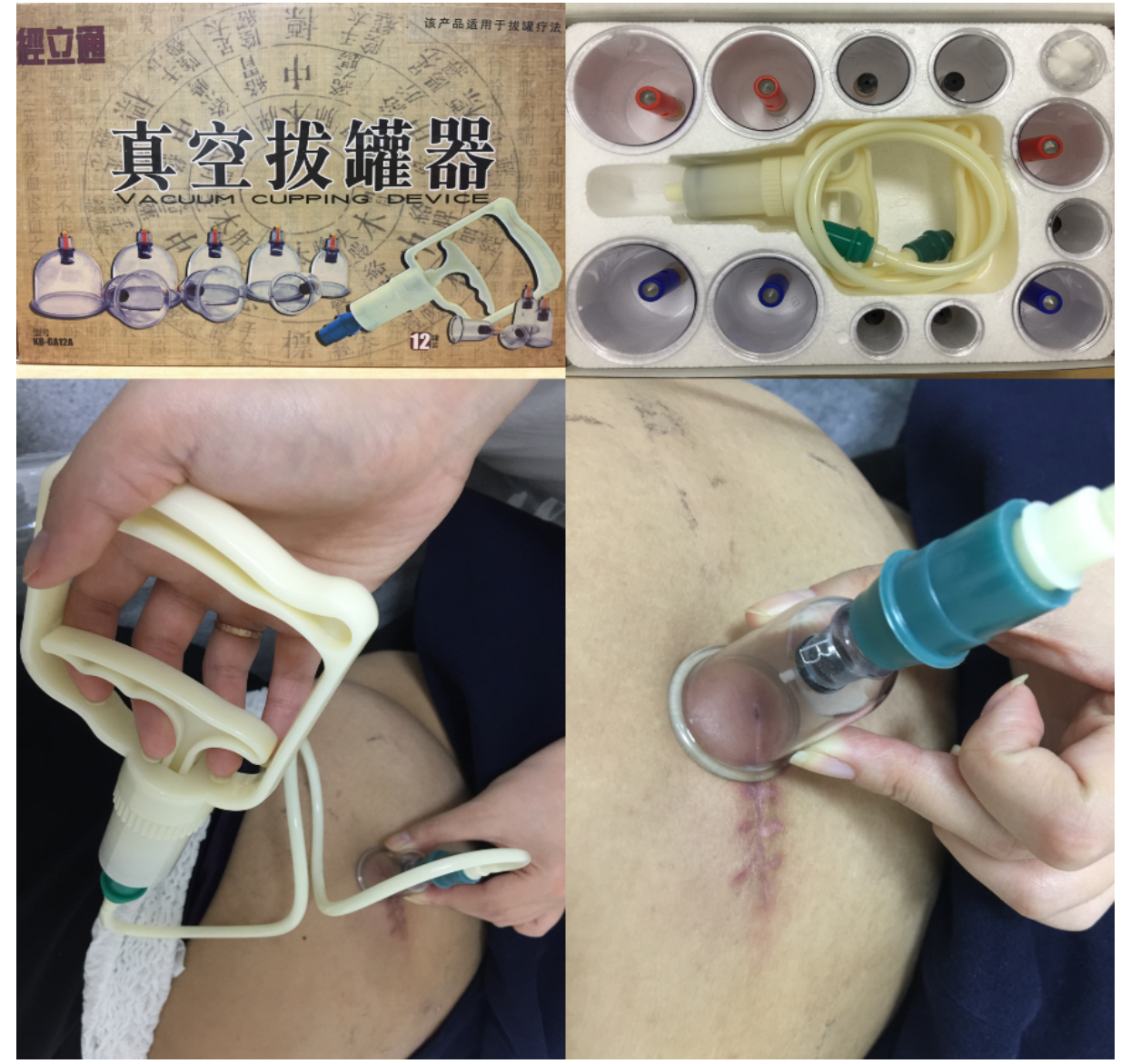

Figure 1: Chinese traditional cupping to suck the fat liquefaction at stimulator implant site after SNM Stage II surgery.

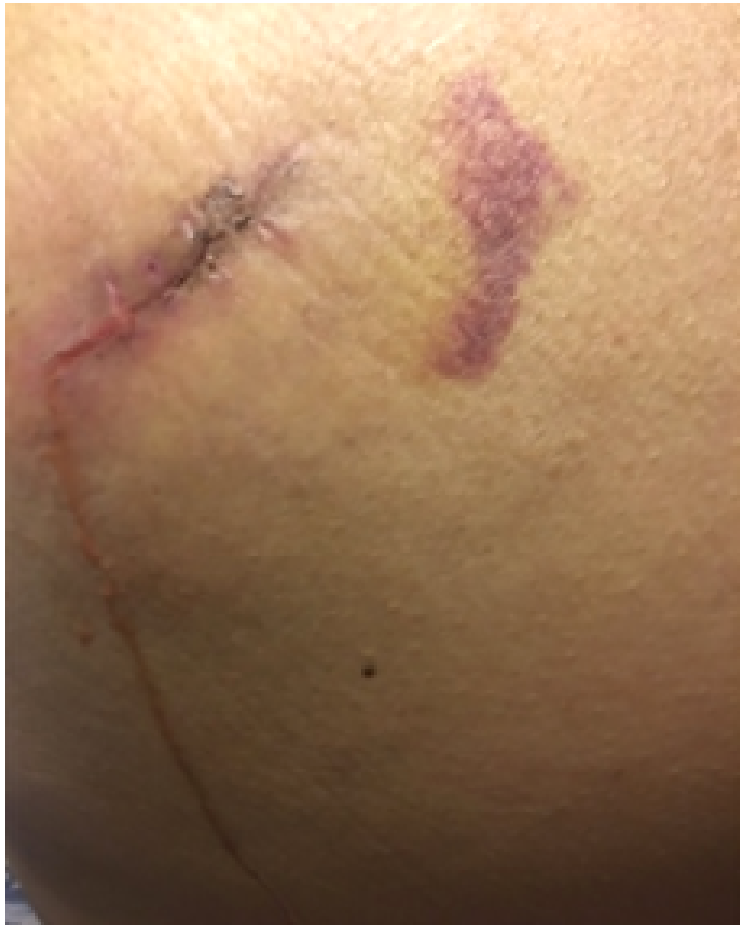

Figure 2: On the seventh postoperative day of the stage II surgery, the wound tissue at the stimulator position was liquefied and broken.

tion, while the liquefaction tracked to the S3 foramen along the wire. Above the $\mathrm{S} 3$ foramen, the lead placement site had some swelling. Ultrasound showed pro- gressively reduced liquefaction at stimulator position but increased liquefaction around the puncture area with swelling. The patient was started on oral antibiotics. Three days later, the fat liquefaction finally resolved and ultrasound showed progressively reduced liquefaction both at the stimulator position and around the lead entry site. She was regularly followed up by ultrasound and we measured the depth of liquefaction area around both the stimulator position and puncture area to note the trend of the change of fat liquefaction (Figure 3).

\section{Discussion}

Sacral neuromodulation (SNM) was developed in the early 1980 s by Tanagho and Schmidt and has become a well-established and widely accepted treatment for patients with refractory lower urinary tract dysfunction, i.e. urgency-frequency syndrome, urgency incontinence, and chronic urinary retention, as well as for patients with bowel dysfunction [4]. In recent years more patients have chosen SNM to treat pelvic diseases and achieve good results. A Meta-analysis with 26 independent studies indicated that SNM might be effective and safe for the treatment of patients with neurogenic lower urinary tract dysfunction [5]. Naseem Mirbagheri's review also showed that SNM improved clinical symptoms and reduced the number of incontinence episodes and severity scores in patients with faecal incontinence, in part by improving anorectal physiological function [6]. 


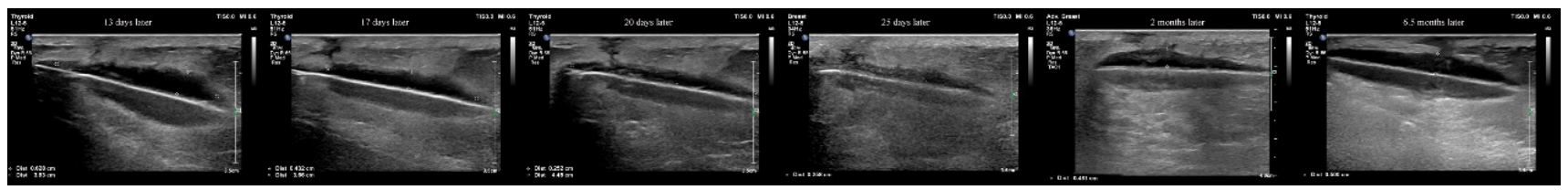

Figure 3: We used ultrasound to monitor the liquid at the stimulator position and measure the distance of the liquid area. The depth of the liquid dark area was reduced from $0.628 \mathrm{~cm}$ to $0.258 \mathrm{~cm}$ within 2 weeks.

Complications with SNM are reported as adverse events that encompass changes in clinical efficacy, pain, wound complications, infection, lead migration, and device failure. Many of these can be managed conservatively but some require surgical intervention [7]. Klaus Bielefeldt's team analyzed adverse events of sacral neuromodulation for fecal incontinence reported to the federal drug administration. Among a total of 1953 patients, complaints led to secondary surgeries in $29.7 \%$ of the adverse events. Reoperations were performed to explant $(38.2 \%)$ or replace $(46.5 \%)$ the device or a lead, or to revise the generator pocket (14.6\%). Conservative management mostly involved changes in stimulation parameters $(44.5 \%)$, which successfully addressed concerns in $35.2 \%$ of cases that included information about treatment results [8].

There are no definitive guidelines that exist for the explantations of sacral nerve stimulators. The International Continence Society recently published a best practice on SNM noting that the IPG should be removed in instances of SNM infection and when the battery was exhausted. (Level of Evidence: III Grade of Recommendation - C) [9]. Re-operation depends on the clinician's empirical judgment. Some types of pain, uncomfortable stimulation, lack or loss of benefit can be improved by reprogramming but a malfunctioning lead, dead battery and other device trouble requires reoperation. In addition, patients with currently available devices are unable to have axial magnetic resonance imaging (MRI) below the head due to concerns about heating of the lead and potential nerve damage [1]. Usually when the skin and subcutaneous tissues at the surgical site become swollen or purulent and skin temperature rises infection is diagnosed. Once this occurs, a systemic antibiotic should be immediately applied. If the infection cannot be controlled sufficiently, complete device removal should be performed. Several studies $[10,11]$ recommend various alternative preoperative prophylactic antibiotics to reduce the incidence of infection.

There are no reports or studies of fat liquefaction of SNM at the surgical site to date. Aseptic fat liquefaction, as a main cause of prolonged healing of aseptic post-surgical incision, is the necrosis of adipose tissue without infection and occurs with an incidence of 0.52 $1.11 \%$ in all postoperative wounds [3]. The mechanism of fat liquefaction is not clear. Incision and drainage is a traditional solution and first-line selection to postoperative wounds infection and liquefaction. Other potential interventions include antibiofilm, microRNA and negative pressure therapy [12-15].

Chinese traditional cupping, with its long history since the Warring States Period ( $5^{\text {th }}$ century BC - 221 BC, is widely used in to treat disease in China. Applications of Chinese traditional cupping may play an anti-inflammatory and analgesic role, facilitate pus discharge and reduce the spread of inflammation secondary to surgery [16]. The mechanism of negative pressure of Chinese traditional cupping is similar to Negative pressure wound therapy (NPWT), which is a sophisticated technique requiring the use of vacuum assisted drainage to remove blood or serous fluid from a wound or operative site [17]. NPWT has been shown to have a positive effect on the healing of infected wounds [18-19]. However, theoretically, the sacral nerve stimulator may migrate under excessive negative pressure which could reduce the therapeutic effect. Thus, Chinese traditional cupping with adjustable negative pressure could be a better alternative to NPWT in those patients who suffer from limited wound infection or fat liquefaction after surgery. Convenience and economic benefits could be other advantages of Chinese traditional cupping rather than NPWT.

\section{Conclusion}

The management of fat liquefaction after SNM may not be limited to re-operation to clear the liquefaction area or removing the stimulator. Given that another invasive procedure means longer recovery periods and a diminished quality of life experience, including the failure of therapy, another less invasive approach to deal with this situation would be of benefit. Our team supports the innovative idea that the use of improved Chinese traditional cupping therapy, a simple kind of NPWT, can help prevent the progression of fat liquefaction. Further research and reports are necessary to confirm our conclusion.

\section{References}

1. Liberman D, MJ Ehlert, SW Siegel (2017) Sacral neuromodulation in urological practice. Urology 99: 14-22.

2. Force Luanne, GD Silva (2017) Management of complications of sacral neuromodulation. Seminars in Colon and Rectal Surgery 28: 173-176.

3. Shi Z, Ma L, Wang H, Yang Y, Li X, et al. (2013) Insulin and hypertonic glucose in the management of aseptic fat liquefaction of post-surgical incision: A meta-analysis and systematic review. International Wound Journal 10: 91-97. 
4. Jens Wöllner, Christian Hampel, Thomas M Kessler (2012) Surgery Illustrated - Surgical Atlas Sacral Neuromodulation. BJU Int 110: 146-159.

5. Thomas M Kessler, David La Framboise, Sven Trelle, Clare J Fowler, Gustav Kiss, et al. (2010) Sacral neuromodulation for neurogenic lower urinary tract dysfunction: Systematic review and meta-analysis. Eur Urol 58: 865-874.

6. Naseem Mirbagheri, Yogeesan Sivakumaran, Natasha Nassar, Marc A Gladman (2016) Systematic review of the impact of sacral neuromodulation on clinical symptoms and gastrointestinal physiology. ANZ J Surg 86: 232-236.

7. Alemi Golnaz, V Dandolu (2013) Sacral neuromodulation therapy of the lower urinary tract: A review of the literature and unanswered questions. Open Journal of Obstetrics \& Gynecology 3: 1-6.

8. Bielefeldt Klaus (2016) Adverse events of sacral neuromodulation for fecal incontinence reported to the federal drug administration. World J Gastrointest Pharmacol Ther 7: 294-305.

9. Howard B Goldman, Jessica C Lloyd, Karen L Noblett, Marcus P Carey, Juan Carlos Castaño Botero, et al. (2018) International Continence Society best practice statement for use of sacral neuromodulation. Neurourol Urodyn 37: 1823-1848.

10. Allen M Haraway, J Quentin Clemens, Chang He, Cynthia Stroup, Humphrey O Atiemo, et al. (2013) Differences in sacral neuromodulation device infection rates based on preoperative antibiotic selection. Int Urogynecol J 24: 20812085.

11. Calvin Lee, Javier Pizarro-Berdichevsky, Marisa M Clifton,
Sandip P Vasavada (2017) Sacral neuromodulation implant infection: Risk factors and prevention. Curr Urol Rep 18: 16.

12. Jing-Chun Zhao, Chun-Jing Xian, Jia-Ao Yu, Kai Shi, Lei Hong (2015) Hypertonic glucose combined with negative pressure wound therapy to prepare wounds with Pseudomonas Aeruginosa infection for skin grafting: A report of 3 cases. Ostomy Wound Manage 61: 28-44.

13. Cooper RA, T Bjarnsholt, M Alhede (2014) Biofilms in wounds: A review of present knowledge. J Wound Care 23: 570-572.

14. Jaideep Banerjee, Yuk Cheung Chan, Chandan K Sen (2011) Micro RNAs in skin and wound healing. Physiol Genomics 43: 543-556.

15. Chenyu Huang, Tripp Leavitt, Lauren R Bayer, Dennis $P$ Orgill (2014) Effect of negative pressure wound therapy on wound healing. Curr Probl Surg 51: 301-331.

16. Jin C, Guangqi Zhang (1988) Clinic usage on cupping therapy. Journal of Traditional Chinese Medicine (Chinese) 7: 63-64.

17. Thomas $S$ (2002) An introduction to the use of vacuum assisted closure. World Wide Wounds.

18. Allen Gabriel, Jaimie Shores, Cherrie Heinrich, Waheed Baqai, Sharon Kalina, et al. (2010) Negative pressure wound therapy with instillation: A pilot study describing a new method for treating infected wounds. Int Wound $\mathrm{J} 5$ : 399-413.

19. Baharestani MM, A Gabriel (2011) Use of negative pressure wound therapy in the management of infected abdominal wounds containing mesh: An analysis of outcomes. Int Wound J 8: 118-125. 